XXX. Kongress der Schweizerischen Gesellschaft für Dermatologie und Venereologie vom 9. und 10. Oktober 1948 in Basel

Dermatologica. 1949;98:255

\title{
A propos de l'effet précoce des rayons X sur la cellule
} épithéliale

\begin{tabular}{|l|l|l|}
\hline W. & Jadassohn \\
\hline E. & & Bujard \\
\hline R. & Paillard \\
\hline P. & Gaudin \\
\hline
\end{tabular}

Travail de la clinique universitaire de Dermatologie (Directeur: Prof. W. Jadassohn) et de l'Institut d'Histologie et d'Embryologie (Directeur: Prof. E. Bujard) de Genève

L'effet précoce des rayons X sur la cellule épithéliale a été examine en employant le Nipple-Test local avec colchicine: tétine de cobaye traitée avec un æstrogène en application local percutanée pour former une acanthose; injection de colchicine avant $\Gamma$ excision de la tétine pour mettre en evidence les mitoses (reaction de Dustin). Nous avons applique $2400 \mathrm{r}(0,5 \mathrm{~mm}$. aim., $5 \mathrm{~mA}$, $70000 \mathrm{~V}$, champ $1,5 \mathrm{~cm}$. de diamètre, distance foyer-peau $24 \mathrm{~cm}$., temps d'irradiation 45' 43"). La reaction de Dustin, très positive dans les tétines de contrôle, est negative $33 \mathrm{~h}$. et demie après Tirradiation; elle est encore negative après 10 jours, mais elle est de nouveau positive après 30 jours.

L'acanthose apparaît, bien que la reaction de Dustin soit negative. Les cellules formant l'acanthose montrent des alterations qui ne se retrouvent plus après 30 jours.

(A paru in extenso in Dermatologica 97, Suppl. 1948.) 